\title{
Economic principles for resource allocation decisions at national level to mitigate the effects of disease in farm animal populations
}

\author{
K. S. HOWE ${ }^{1 *}$, B. HÄSLER ${ }^{2}$ AND K. D. C. STÄRK ${ }^{2}$ \\ ${ }^{1}$ Centre for Rural Policy Research, College of Social Sciences and International Studies, University of Exeter, \\ Devon, $U K$ \\ ${ }^{2}$ Veterinary Clinical Sciences, Royal Veterinary College, North Mymms, Hatfield, UK
}

Received 13 September 2011; Final revision 28 January 2012; Accepted 12 March 2012; first published online 23 April 2012

\section{SUMMARY}

This paper originated in a project to develop a practical, generic tool for the economic evaluation of surveillance for farm animal diseases at national level by a state veterinary service. Fundamental to that process is integration of epidemiological and economic perspectives. Using a generalized example of epidemic disease, we show that an epidemic curve maps into its economic equivalent, a disease mitigation function, that traces the relationship between value losses avoided and mitigation resources expended. Crucially, elementary economic principles show that mitigation, defined as loss reduction achieved by surveillance and intervention, must be explicitly conceptualized as a three-variable process, and the relative contributions of surveillance and intervention resources investigated with regard to the substitution possibilities between them. Modelling the resultant mitigation surfaces for different diseases should become a standard approach to animal health policy analysis for economic efficiency, a contribution to the evolving agenda for animal health economics research.

Key words: Infectious disease control, infectious disease epidemiology, health economics, surveillance, veterinary epidemiology.

\section{INTRODUCTION}

Animal disease is an economic problem with veterinary implications, not a veterinary problem with economic implications, because it affects people's wellbeing. The most evident manifestation is lost production. This translates, for example, into reduced availability of meat, milk, or eggs wanted for human consumption, depleted capital stock for further production because breeding animals are culled or die prematurely, and

\footnotetext{
* Author for correspondence: Dr K. S. Howe, Room 302, Laver Building, North Park Road, University of Exeter, Devon, EX4 4QE, UK.

(Email: K.S.Howe@exeter.ac.uk)
}

fewer products for further processing before final consumption, such as hides and skins and animal byproducts used in pharmaceuticals manufacture. All of these physical losses can be expressed in monetary units, and aggregated as one measure of lost value to society.

Diminished animal welfare, partly a by-product of disease, also affects people adversely because of their empathy with other sentient beings. Similarly, people's fear of zoonoses as well as actual harm to their physical health, exemplified by bovine spongiform encephalopathy (BSE), also reduces well-being. Expressing these sources of lost wellbeing as monetary values is very difficult, currently impossible in some 
instances, but they are nevertheless real. They must be quantified, if possible, and always taken into account for animal health policy. In the longer term, it may be possible to devise more techniques based on willingness-to-pay approaches, like contingent valuation, that enable monetary values for such wider economic benefits to be measured.

Animal disease is therefore 'bad' for society and its effects need to be mitigated. European Union (EU) countries have adopted strategies based on the notion that 'prevention is better than cure' $[1,2]$. But, as will be shown, this is not unequivocally true. Avoiding losses gives rise to costs incurred by disease mitigation. These include resource expenditures for research, the design and investment in measures for surveillance and intervention, and their implementation by qualified personnel. All such resources have opportunity costs, the benefits foregone as a result of allocating them to disease mitigation instead of other productive use. Thus, before any decisions are made, there is need for close scrutiny of the implications for economic efficiency of assumptions that underpin disease mitigation policy. The economics of animal health is a branch of applied microeconomics, its closest relation agricultural production economics. With its central proposition that decisions are made subject to technical constraints, the principles rooted in Heady's classic work [3] and updated by Beattie et al. [4] are an indispensable guide to the variables and relationships that should be defined before any attempt is made to quantify by mathematical modelling or empirical analysis.

This paper originated in a project for the Swiss Federal Veterinary Office, with the objective of developing a practical, generic tool for the economic evaluation of animal disease surveillance programmes that are part of the National Control Plan [5]. Experience from this particular context is therefore potentially relevant for decision making in other countries. The scope and limitations of the approach in international perspective are reviewed in our conclusions. The research proceeded on two premises. First, economic logic is paramount. Applied problem analysis must be based on robust economic principles. In other words, any quantitative model must always be founded on a sound theoretical model. Second, the principles should help policy-makers think more logically about the specific role of surveillance in disease mitigation, and the wider implications for resource allocation decisions. Importantly, this relates to the decision-making process itself. Choices made about the analytical techniques employed, including the use of sophisticated mathematical or econometric procedures, are secondary issues. In practice, such choices are constrained by the nature of a particular decision problem, access to data, and the time available for analysis.

The principles are explained as follows. First, disease mitigation, defined as loss reduction achieved by surveillance and intervention, is interpreted from an epidemiological perspective, and then as corresponding economic relationships. The example is for epidemic disease, but the principles similarly apply to endemic disease. Second, the implications of attributing economic values for mitigation policy decisions are illustrated. Third, and very importantly, it is shown that to consider surveillance independently of intervention in mitigation policy is meaningless in economic terms. The relationships between surveillance, intervention and avoided losses must be considered simultaneously. This leads to the concept of a mitigation surface, a template to guide decisionmaking for economic efficiency in disease mitigation. In conclusion, suggestions are made as to how the Swiss project experience adds to current ideas for advancing a research agenda with the potential to help policy-makers for disease mitigation at national level make better informed decisions.

\section{EPIDEMIOLOGY AND ECONOMICS IN DISEASE MITIGATION}

For reasons that will become apparent, the analysis begins with consideration of the implications of disease for farm production of conventional livestock products. Economic production decisions are made subject to technical constraints, so the technical relationship between physical losses in livestock production and the process of disease mitigation is of fundamental importance. For epidemic disease, incidence is the critical variable, and the relevant basic concept the epidemic curve [6]. New cases occurring throughout the period of a disease event have a cumulative effect on aggregate production. Depending on the specific disease, physical production losses may include reduced current outputs of, say, eggs or milk, and reduced capacity for future production because of abortions or breeding stock mortality. All such physical losses can be aggregated in monetary terms, transforming an epidemic curve into a lost value of production curve. The latter curve shows the additional, or marginal, value of lost production which 


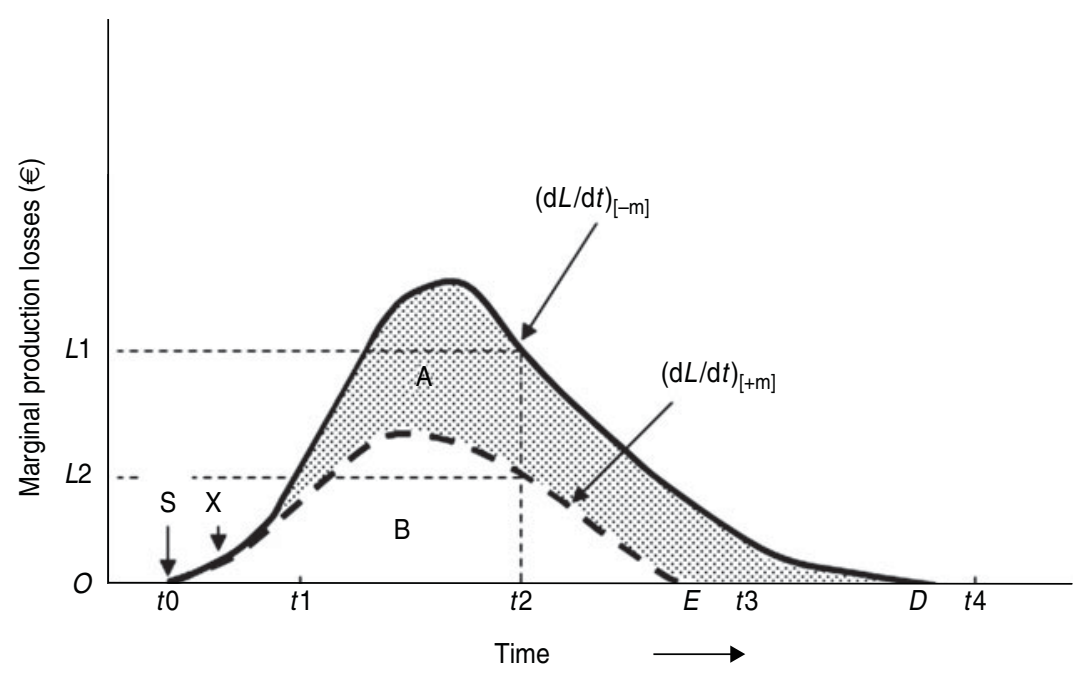

Fig. 1. Economic implications of epidemic disease, with and without mitigation.

results from the new cases occurring at each point in time as an epidemic proceeds. Figure 1 is a stylized illustration of the relationship.

\section{THE TIME PATH OF PRODUCTION LOSSES}

It is assumed that the first cases of disease are observed at time $t 0$. Thereafter, new cases and attendant losses accumulate until, at $t 2$ for example, with the epidemic past its peak, they add OL1 to the monetary value of lost production. Similar interpretations apply to all other points along curve $S D$, where $S$ corresponds with $t 0$, labelled $(\mathrm{d} L / \mathrm{d} t)_{[-\mathrm{m}]}$, signifying that it shows the marginal addition to losses with respect to time without mitigation.

Curve $S D$ is the benchmark for the economic appraisal of any disease mitigation programme because, mathematically, the integral of curve $(\mathrm{d} L / \mathrm{d} t)_{[-\mathrm{m}]}$ over the range $S D$, equal to area $A+B$, is the total monetary value of all production irretrievably lost if an epidemic is left to run its natural course. Epidemiological modelling can make a crucial contribution to economic analysis by providing estimates for the physical production losses expected from uncontrolled epidemics, then to be translated into monetary values. Such losses typically include animals lost due to mortality, reduced growth of animals, reduced production (e.g. less milk, wool, eggs) or reduced reproduction (e.g. because of abortion, smaller litters).

Curve $S E$ traces marginal production losses with mitigation, $\mathrm{d} L / \mathrm{d} t_{[+\mathrm{m}]}$. It, too, is a subject for epidemiological modelling. Its origin is shown at $t 0$, assuming that mitigation begins immediately the first cases of a developing epidemic are observed. Over the range $S$ to $X$, identical curves with and without mitigation indicate an assumed lagged response to the first beneficial effects of mitigation. Only to the right of $X$ does mitigation begin to limit production losses. For example, at $t 2$ marginal production losses from new cases are reduced from OL1 without mitigation to OL2 with mitigation.

It follows that area $B$ below curve $S E$, the integral of $(\mathrm{d} L / \mathrm{d} t)_{[+\mathrm{m}]}$ over range $S$ to $E$, is the total monetary value of production losses with disease mitigation. If $S E$ corresponds to the epidemic curve having applied the best available technical approach to mitigation, the area between curves $S D$ and $S E$, labelled $A$ in Figure 1, represents the maximum value of avoidable production losses feasible with existing mitigation procedures. Curve $S E$ is therefore a technical efficiency frontier. The closer is $S E$ to the horizontal axis, the greater the technical efficiency of mitigation. Its location depends on scientific knowhow and mitigation technology, as well as characteristics of the relevant livestock sector including factors such as the population density of herds or flocks, farm husbandry and management efficiency, the natural environment and, not least, the real resources of personnel and equipment available to implement mitigation policy when needed. Sometimes disease eradication is technically possible (e.g. rinderpest worldwide), and sometimes not. In relation to Figure 1, the relevant economic question is by what proportion area $A$ should be reduced to maximize social benefit. This will be addressed in the following sections. 


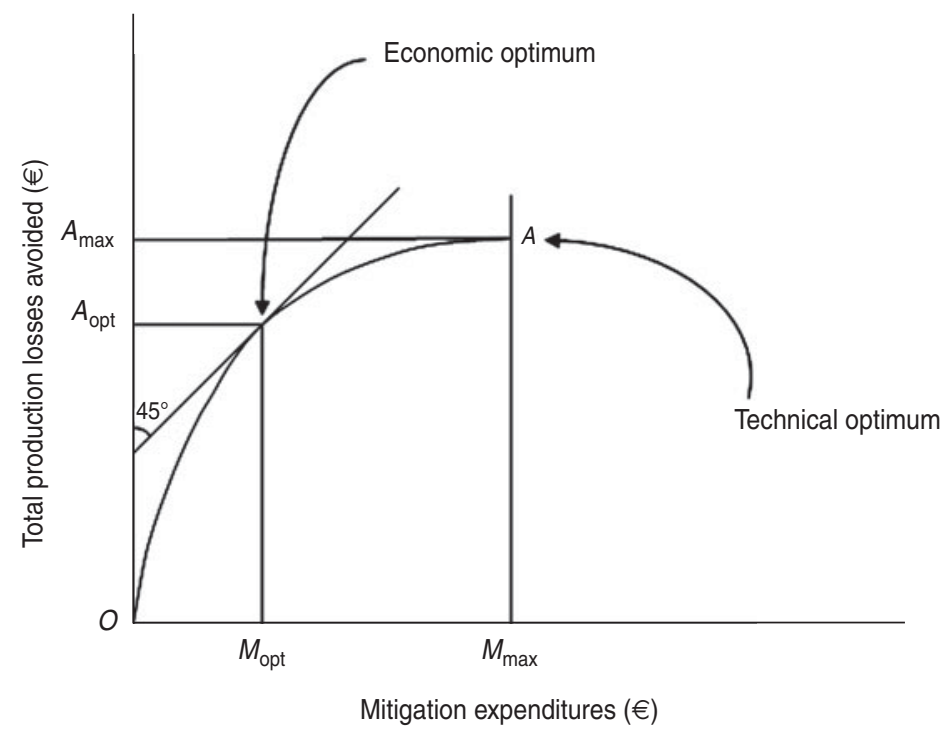

Fig. 2. Optimal economic efficiency is not the same as optimal technical efficiency in disease mitigation.

\section{Optimal resource use for disease mitigation}

Avoiding production losses requires expenditures on mitigation resources. First, there must be an investment of fixed resources to provide an infrastructure of laboratories for scientific research, the development of technologies to combat specific diseases, and a basic capacity of personnel and equipment sufficient to mobilize effort to combat the effects of disease. To justify fixed investment in economic terms requires that the benefits accruing over time are estimated as at least sufficient to cover their cost, an assessment based ideally on the outcome of formal social benefit-cost analysis. Second, responding to the perceived consequences of any specific disease, whether emerging, endemic, or epidemic such as outlined above, also requires consideration of the need for further resource expenditures specific to it. Importantly, such variable resources for mitigation effort are normally expected to exhibit diminishing returns, a widely observed phenomenon of the real world. This means that the closer physical production losses avoided approach the maximum technically possible the more difficult, and therefore costly, it is to expunge the residual.

In all such situations, the focus of the present research, optimal economic efficiency, or maximum net benefits, is found where the marginal benefits from production losses avoided are just sufficient to cover the marginal mitigation costs of obtaining them. Marginal mitigation costs, or expenditures on resource provision, are the mathematical product of quantities of real resources used and their respective money prices. Conceptually, individual resources can be viewed as adding up to a single aggregate resource, its price an index of all individual input prices weighed by their respective contributions to the aggregate unit of resource. Identical logic applies to different physical products which are the individual components of production losses avoided. Mathematically, optimal economic efficiency of mitigation is defined as

$P_{\mathrm{A}} \cdot \mathrm{d} A=P_{\mathrm{M}} \cdot \mathrm{d} M$,

where $P_{\mathrm{A}}=$ monetary value of an increment of physical production losses avoided (benefit); $\mathrm{d} A=$ increment of physical production losses avoided; $P_{\mathrm{M}}=$ monetary value of an increment of mitigation resources (cost); $\mathrm{d} M=$ increment of real resources used for disease mitigation.

In relation to Figure 1, the criterion identifies by how far mitigation activities need to move curve $S D$ in the direction of curve $S E$ to maximize net benefits. Aiming to reach curve $S E$, optimizing the technical efficiency of mitigation, is justified only if mitigation is costless, which is unrealistic, or if the benefits relative to mitigation costs are extraordinarily high. This may be so if potentially catastrophic effects ('negative externalities') of zoonotic diseases on human populations, as is the case with BSE or avian influenza, are also to be avoided. Figure 2 illustrates the implications of the relationship in conventional economic terms.

Figure 2 relates the total monetary value of production losses avoided, $A$, for different total levels of mitigation resource expenditures, $M$, necessary to achieve them. Curve $O A$ is therefore a mitigation production function, $A=f(M)$, fundamentally a 
technical relationship but with variables expressed in monetary units, here euros $(€)$. On the vertical axis, the monetary values for losses avoided correspond to increasing proportions of area $A$ in Figure 1. Because both $A$ and $M$ are in monetary units, a $45^{\circ}$ tangent to the mitigation function from the vertical axis identifies the combination of avoided production losses and mitigation expenditures that maximizes net benefits. At that point, an additional $€ 1000$, say, of mitigation expenditure is recouped by an additional $€ 1000$ of avoided losses. Anywhere to the left, the marginal value of avoided losses always exceed marginal mitigation expenditures; to the right, marginal losses avoided are always less than marginal mitigation expenditures. Only at coordinates $A_{\text {opt }}$ and $M_{\text {opt }}$ are net benefits - total avoided production losses less total mitigation costs - maximized.

Rearranging equation (1) as

$\frac{\mathrm{d} A}{\mathrm{~d} M}=\frac{P_{\mathrm{M}}}{P_{\mathrm{A}}}$

separates the technical relationship from the monetary values. It highlights that the economic optimum depends on three variables, $(a)$ the technical production function relating production losses avoided to mitigation effort, $(b)$ the monetary expenditure for a unit of mitigation resources, $P_{\mathrm{M}}$, and (c) the monetary benefit from avoiding a unit of production losses, $P_{\mathrm{A}}$. Thus the economic optimum level for mitigation effort will change for different mitigation technology, any variation in the value obtained from avoided losses relative to the value foregone from expending mitigation resources to reduce losses, or both. For example, better scientific understanding of disease epidemiology, improved vaccines, and other measures to limit infection spread, cause curve $O A$ in Figure 2 to pivot upwards about the origin. In other words, the appliance of science increases the productivity of real mitigation resources. Increased mitigation effort is now justified to avoid more production losses.

If mitigation effort is to exceed the economic optimum with respect to the monetary value of production losses avoided, both monetary and non-monetary values obtained from other benefits must be taken into account. Examples include the notional value society attaches to avoiding epidemic disease becoming endemic, or the fear of a zoonosis, such as BSE or avian influenza virus, considered to pose an unacceptable level of risk to people, as well as expenditures for treating consequential human illness that can be estimated. Defining $P_{\mathrm{A}+}$ as the monetary value of conventional production losses augmented by these other benefits, in the limit ratio $P_{\mathrm{M}} / \mathrm{P}_{\mathrm{A}}+$ may be so small (i.e. tends to zero) that maximum mitigation effort is indeed justified (where $\mathrm{d} A / \mathrm{d} M$ tends to zero). Then point $A$ in Figure 2, coordinates $A_{\max }, M_{\max }$, is both a technical and an economic optimum, and $\left(M_{\max }-M_{\mathrm{opt}}\right)$ is the minimum value implicitly attributed to the benefits arising from other than production losses avoided as a result of maximizing mitigation effort. Crucially, this logic justifies 'prevention is better than cure'. But it cannot be emphasized too strongly that for diseases without such extreme detrimental effects, or negative externalities, prevention is not justified in economic terms except in the wholly unrealistic circumstance that mitigation resources are free.

Strictly, equation (2) is an insufficient condition for overall economic efficiency. Two criteria apply. Respectively, these concern the least cost combinations of surveillance and intervention resources, and the scale at which surveillance and intervention resources are combined at least cost to maximize net benefits.

\section{MITIGATION, SURVEILLANCE AND INTERVENTION}

In the real world, mitigation comprises two conceptually distinct activities, surveillance $(S)$ and intervention $(I)$, each with its particular endowment of resources, both contributing to loss avoidance. The modified mitigation function becomes

$A=f(M)=f(S, I)$.

Surveillance is the systematic ongoing collection, collation, and analysis of data related to animal health, objectively to inform decisions for the mitigation of public health hazards, and to demonstrate the absence of disease, infection, or foodborne hazards. Intervention is the process of implementing measures directed at mitigation. Logically, surveillance informs decisions made about the nature and scope of interventions, or how existing interventions should be changed in the light of new information. Intuitively, there is a trade-off between the two. More and better surveillance information should facilitate better targeted and timely intervention. Surveillance and intervention are probably economic substitutes, i.e. the more resources expended on surveillance, the fewer resources should be needed for intervention, or vice versa. Whether they are in fact depends on the technical relationship between them, an empirical 
issue, the ramifications of which are now discussed. Taking into account their relative costs of provision, the objective is to identify combinations of $S$ and $I$ that minimizes the cost of any corresponding level of avoided losses, $A$.

\section{LEAST-COST SURVEILLANCE AND INTERVENTION}

For reasons analogous to the explanation for equation (1), a least-cost combination of $S$ and $I$ is found uniquely where $P_{\mathrm{S}}$.d $S=P_{\mathrm{I}}$.d $I$. Any other outcome costs more, inequality indicating that the cheaper of $S$ and $I$ should be substituted for the more expensive.

Rearranging terms,

$\frac{\mathrm{d} S}{\mathrm{~d} I}=\frac{P_{\mathrm{I}}}{P_{\mathrm{S}}}$,

where $\mathrm{d} S / \mathrm{d} I=$ rate of technical substitution of $I$ for $S$; $P_{\mathrm{I}} / P_{\mathrm{S}}=$ monetary cost of providing $I$ relative to $S$.

The technical relationship $\mathrm{d} S / \mathrm{d} I$ is very important, its full significance apparent from a related parameter, $\sigma$, the Hicks elasticity of substitution of $I$ for $S$, 'a measure of the ease with which the varying factor can be substituted for others' $[7,8]$.

Mathematically,

$\sigma=[\mathrm{d}(S / I)(S / I)][(\mathrm{d} S / \mathrm{dI}) \mathrm{d}(\mathrm{d} S / \mathrm{d} I)]$

is the proportional change in the ratio of $S$ to $I$ resource use relative to the proportional change in rate of technical substitution of $I$ for $S$. Its magnitude can vary between zero and infinity. Its economic importance is best illustrated from an iso-mitigation map.

\section{Iso-mitigation maps}

An iso-mitigation map plots variable combinations of $S$ and $I$ that give rise to different fixed levels of $A$. Its characteristics depend exclusively on the technical relationship between $S, I$, and $A$ in any particular case. Figure 3 is a stylized illustration of the implications of different values of $\sigma$ for least-cost mitigation for two ratios for the monetary cost of providing $S$ to relative to $I$. The total budget available to finance resource provision increases with distance from the origin. The specifics of such relationships require careful consideration for modelling or empirical analysis, including whether $\sigma$ is constant for all levels of $A$, or perhaps varies systematically with increasing values of $A$ as shown here.

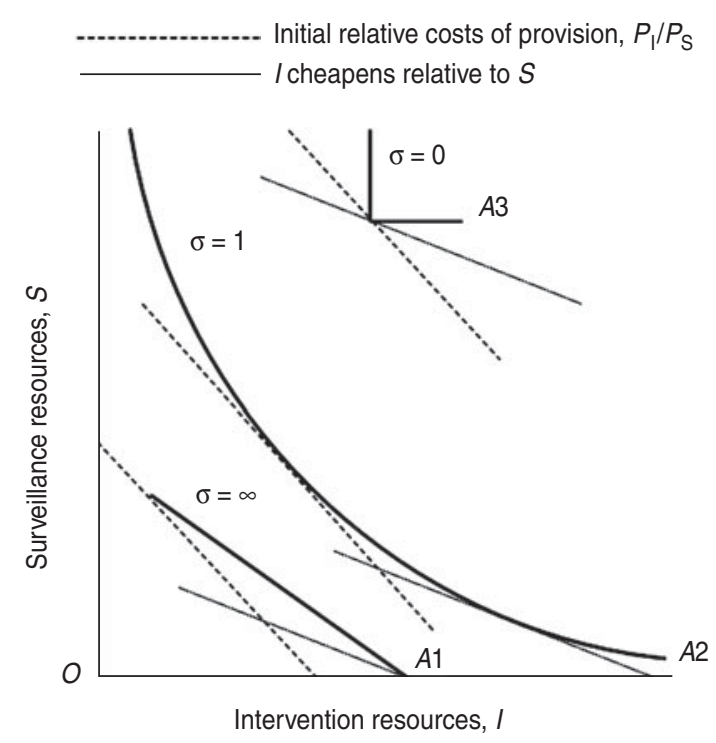

Fig. 3. Implications of the Hicks elasticity of substitution, $\sigma$, for mitigation resource allocation under two sets of relative costs of providing surveillance, $S$, and Intervention, $I$; Iso-mitigation curves for avoided production losses, $A 3>A 2>A 1$.

Figure 3 shows $\sigma$ diminishing from $\infty$ to 0 for increasing magnitudes of $A$. For $\sigma=\infty$, equation (4) implies that either all $S$, or all $I$, will account for mitigation depending on which is the cheaper option; $S$ and $I$ are described as perfect substitutes. But in the present context, for reasons explained below, some minimum provision for intervention is necessary. For $\sigma=0, S$ and $I$ must be used in fixed proportions, because substitution is impossible irrespective of changes in relative costs of provision; $S$ and $I$ are described as perfect complements, and must be regarded as one resource, $M$. For $\sigma=1$, the less expensive resource substitutes for the relatively more expensive. In general, the greater the magnitude of $\sigma$ the more sensitive is the least-cost solution for budget allocation between $S$ and $I$ for changes in their relative costs of provision. Arguably, disease mitigation policies in practice are typically formulated as if $\sigma=0$, if only because few discrete options for combining $S$ and $I$ are ever considered.

\section{The overall economic optimum for disease mitigation}

Overall economic optimization for disease mitigation requires that the least-cost combination of $S$ and $I$ is for that level of $A$ where net benefits are maximized.

From equation (2),

$\frac{\mathrm{d} A}{\mathrm{~d} M}=\frac{P_{\mathrm{M}}}{P_{\mathrm{A}}}$, 


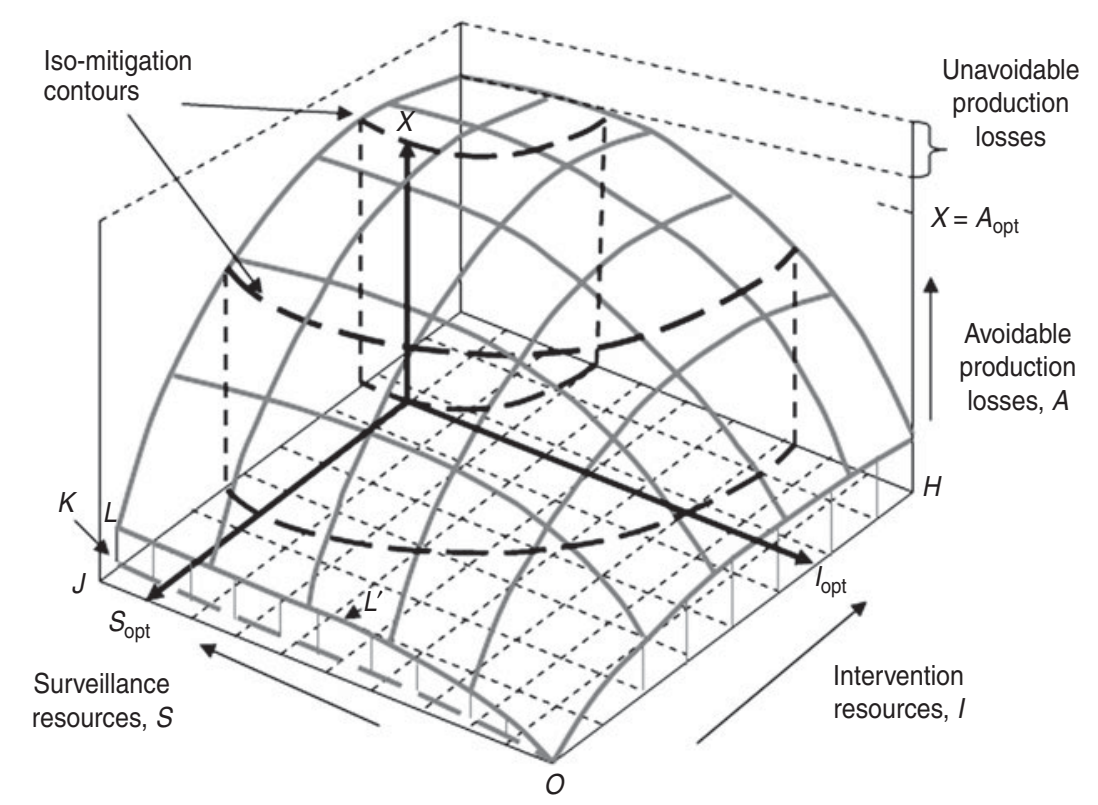

Fig. 4. Hypothetical epidemic disease mitigation surface, diminishing returns to $S$ and $I, 1<\sigma<\infty$.

so that

$P_{\mathrm{A}} \frac{\mathrm{d} A}{\mathrm{~d} M}=P_{\mathrm{M}}$

In words, the marginal value product of mitigation in terms of avoided production losses equals the monetary cost of providing a unit of mitigation resources. Since $M$ comprises $S$ and $I$,

$\frac{\mathrm{d} A}{\mathrm{~d} M}=\frac{\partial A}{\partial S} \mathrm{~d} S+\frac{\partial A}{\partial I} \mathrm{~d} I$,

where $\partial A / \partial S$ and $\partial A / \partial I$ are the marginal physical products of $S$ and $I$ associated with increments of $\mathrm{d} S$ and $\mathrm{d} I$. Thus following equation (6), we require that for given monetary values of $P_{\mathrm{S}}$ and $P_{\mathrm{I}}$

$P_{\mathrm{A}} \frac{\partial A}{\partial S}=P_{\mathrm{S}}$

and

$P_{\mathrm{A}} \frac{\partial \mathrm{A}}{\partial \mathrm{I}}=P_{\mathrm{I}}$

are solved simultaneously for $S, I$, and $A$. This amounts to searching across an iso-mitigation map until the level of $A$ is reached at which both $S$ and $I$ are in least-cost combination and the total monetary value of losses avoided (total benefits) minus the monetary cost of surveillance and intervention (total costs) is maximized.

\section{THE DISEASE MITIGATION SURFACE}

The relationships above have practical implications for the planning and design of national surveillance and intervention programmes. From an economics perspective, $S$ and $I$ need to be considered together, not independently. An iso-mitigation curve is a contour line on a three-dimensional relationship, namely a disease mitigation surface with characteristics determined by equation (3). Figure 4 is a hypothetical example for epidemic disease, drawn to incorporate what are expected to be typical features, especially diminishing returns to both $S$ and $I$. It illustrates why acquiring knowledge of disease-specific characteristics of actual surfaces by modelling and empirical research is indispensable to the design of mitigation policies for optimal economic efficiency.

Figure 4 shows that along axis $O H$, intervention increasing without surveillance, production losses are nevertheless avoided. But if any given level of $I$ is supplemented by surveillance, i.e. in the direction $O J$, more production losses are avoided. There are technical benefits from combining the two mitigation elements. However, the interpretation for axis $O J$, increasing surveillance without intervention, is different. Surveillance always requires some provision for intervention, however small, because the immediate response to any disease incidence identified by surveillance must be to trigger activities aimed at inhibiting its progress. Thus the relevant lower boundary of the 
production surface with respect to surveillance corresponds to $O K$, a curvilinear relationship on the horizontal plane tracing out the path of increasing avoided production losses, $O L$, for the lowest feasible levels of intervention consistent with any loss avoidance. Intervention therefore limits the scope for increasing surveillance to contribute any further to loss avoidance beyond point $L^{\prime}$, where $\partial A / \partial S$ becomes zero, and similarly for curves associated with higher levels of intervention.

Another feature of the surface is flattening towards its apex, indicating the assumed impossibility of eliminating all production losses. Surveillance and intervention have zero marginal products at high levels of resource use. But for any such estimated mitigation surface, the most important economic information derives from solving equations (8) and (9). This defines the coordinates for production losses avoided and associated levels of surveillance and intervention resource use that optimize net benefits, shown here as $A_{\text {opt }}, S_{\text {opt }}$ and $I_{\text {opt }}$. Any change in relative monetary values changes the optimal location on a given mitigation surface. Similarly, and consistent with the discussion of Figure 2, a change in mitigation technology alters the mitigation surface itself. An improvement represents increased productivity of mitigation resources, causing a vertical shift in the surface. The precise effect on the surface topography depends on whether the improved technology originates from better surveillance methods, intervention methods, or both.

\section{TOWARDS A NEW RESEARCH AGENDA}

In recent years, animal health economics has increasingly been a focus for discussion about new research directions [9-12]. These stress, for example, the need to adopt a New Institutional Economics approach, and to base economic and epidemiological analysis on value chain concepts. Crucially, all perspectives recommend taking a more holistic view of the implications of problems in animal health. Animal disease has adverse repercussions for people's wellbeing far beyond the individual farmer or livestock keeper, affecting entire regions, countries, international trade, and causing scarce resources to be diverted away from other sectors of economic activity. As Rich \& Perry show [11], developing countries present especially complex problems, particularly with regard to the nature of incentives to effect change in the different socioeconomic contexts that determine people's behaviour, and for acquiring data. A much broader social sciences approach than just economics will be needed to accommodate the variability of decisionmaking contexts encountered in such countries.

This paper, however, is concerned specifically with strategic national decision-making, and for a developed market economy. The basic infrastructure of physical and human capital to address problems of disease mitigation is in place, and planning resource allocation therefore tends to focus on the estimated costs and benefits of emerging diseases, confronting epidemic disease outbreaks, or reducing endemic disease prevalence. It follows that the standard microeconomic principles set out in this paper are a robust, logical and practical foundation for decisions aimed at improving the economic efficiency of policies to mitigate the effects of animal disease. They are a basis for applied quantitative analysis, and can provide new perspectives on the technical and economic dimensions of disease mitigation. However, on the evidence available, conceptualizing disease mitigation as outlined above explicitly as a three-variable process is novel. Even with the resource infrastructure already available, much needs to be done before benchmark mitigation surfaces can be estimated. Planning is a forward looking or ex-ante activity. The empirical analyses that underpin the Switzerland project showed that mainly only ex-post appraisal of decisions already taken was possible [13-15]. Moreover, benefit-cost or cost-effectiveness analysis were the only evaluation methods currently feasible.

Benefit-cost analysis is a widely employed approach to the analysis of investment decisions, especially for the public sector, as in the present context, where resource expenditures and resultant benefits accrue over time. Its superficial simplicity obscures complex theoretical issues that continue to be the object of attention [16-18]. However, a more fundamental issue is of concern here, its full implications explored in the literature by McInerney et al. [19, 20] and especially Tisdell [21]. A benefit-cost ratio is strictly an acceptability criterion, not an optimizing criterion. Unlike the principles here, which emphasize the crucial role of marginal criteria in defining optimal efficiency, a benefit-cost ratio $(\mathrm{B} / \mathrm{C})$ is an average. If greater than unity, it tells us only that in net terms society is better off from a particular policy, not whether there might be some better alternative. Comparison of alternative levels of resource use to mitigate the effects of any given disease on the basis of benefit-cost ratios is misleading when the objective is 
optimal economic efficiency. With reference to Figure 2 , for example, $\mathrm{B} / \mathrm{C}=A / M$ is the slope of any ray drawn from the origin to the mitigation function. Under diminishing returns, low levels of mitigation resource use generate higher $\mathrm{B} / \mathrm{C}$ ratios than do higher levels of resource use. Counter-intuitively, optimal economic efficiency is found where mitigation resources expenditure is relatively high and the $\mathrm{B} / \mathrm{C}$ ratio relatively low. This is not a novel insight, but one commonly overlooked. Crucial to the present argument is the fact that the surveillance and intervention resources constituting $\mathrm{C}$ also must be combined at least cost (Fig. 3), and this is not taken into account.

In short, although a high $\mathrm{B} / \mathrm{C}$ ratio with low mitigation expenditures is intuitively appealing, it may well lead to an incorrect decision with regard to satisfying economic efficiency criteria when investigating options for a given disease. An exception is with increasing returns to mitigation resource use. Whether this is in fact the case is an empirical question, most likely to occur for low levels of variable resource use, and in less developed countries where investment for disease mitigation is constrained by lack of funds. Under increasing marginal returns, maximizing $\mathrm{B} / \mathrm{C}$ is a valid policy objective. Whatever the circumstances, society's objective should always be to optimize economic efficiency, because there are many competing demands on scarce resources. This argument has added weight in periods of financial austerity, such as when government budget cuts exacerbate expenditure constraints on state-funded disease mitigation programmes.

This paper, and the empirical investigations based on its arguments, make evident why research into the specific characteristics of mitigation surfaces for different animal diseases must be a priority. Thus far, social benefit-cost analysis has dominated, because there is no alternative. The economic principles set out here are not new, but need to become an indispensable guide for adoption by analysts and national policy-makers concerned with evaluating mitigation policies in farm animal health. Importantly, we believe that our findings should have organisational consequences. In many national veterinary services, and also at European Union level, there tend to be separate units involved, one for setting animal health policy (including technical surveillance and intervention strategies) and another to determine the economic implications. We argue strongly for closer integration of economic concepts in the early stages of design and planning of animal disease surveillance and control programmes, or the modification of existing schemes.

Crucially, the explicit objective in planning mitigation policy for a given disease should be to acquire the most comprehensive understanding possible of the three-variable relationships, both technical and economic. The steps involved require specification of the relevant disease aetiology, the technological options (e.g. vaccination, test and removal) for implementing mitigation in association with available surveillance procedures, including all relevant personnel, identification of the output and resource variables key to estimating the benefits and costs from feasible combinations, and data assembly for all categories including sources for deriving monetary coefficients. The implications of different technical approaches to surveillance and intervention for resource requirements and economic losses avoided can then be modelled.

In the past, production economics analysis relied on econometric estimation [22, 23]. Now, for animal disease, epidemiological modelling opens up entirely new possibilities to generate mitigation surfaces for economic analysis from veterinary data. At its most sophisticated and comprehensive, this will be mathematical and incorporate risk analysis. But there may be situations where spreadsheet budgeting is deemed sufficient, or all that is possible in a real world policy environment. This will be so when decisions must be taken quickly, or if data are scarce and expert opinion plays a significant role in what, in effect, are 'try it and see' simulation exercises. Whatever techniques are used, the essential analytical perception is that equation (3) $[A=f(M)=f(S, I)]$ is the relationship to be estimated, documented, and investigated for the implications of changes in monetary values and technical options for the consequent costs and benefits that accrue over time.

As a result of integrating economics and epidemiology in the ways described, policy makers may have access to better information on which to base decisions aimed at enhancing the efficiency of resource allocation to improve animal health. In any market economy, developed or less developed, the logic of relating social values of economic products (benefits) and the scarce resources that provide them (costs) to the technical relationships between them (production functions) to maximize people's wellbeing is inviolable. The next stage in implementing the approach is to instruct analysts and policy decision-makers in the Swiss project context. The objective is to identify 
scope for enhancing existing capacities for policy analysis by applying the economic logic set out, as well as to consider the financial implications of any supplementary investments considered necessary. A priori it is expected that closer integration of epidemiological and economic understanding in research is key, and achievable at relatively low cost. Improving the methodology is the critical factor, and this will be fully justified if the value added from efficiency gains in resource allocation for disease mitigation exceeds the associated costs.

\section{ACKNOWLEDGEMENTS}

The financial support of the Swiss Federal Veterinary Office is gratefully acknowledged, also acknowledged are John McInerney for his comments on an earlier draft and at the Black Horse Seminars, and an anonymous referee.

\section{DECLARATION OF INTEREST}

None.

\section{REFERENCES}

1. Department of the Environment, Food and Rural Affairs, Scottish Executive, Welsh Assembly Government. Animal Health and Welfare Strategy for Great Britain. London: Defra, 2004 pp. 40.

2. Health and Consumer Protection Directorate-General. A new animal health strategy for the European Union (2007-2013) where 'prevention is better than cure', Communication from the Commission to the Council, the European Parliament, the European Economic and Social Committee and the Committee of the Regions, COM 539, 2007 final. Brussels : European Commission, 2007, pp. 28.

3. Heady EO. Economics of Agricultural Production and Resource Use, Englewood Cliffs, N.J: Prentice-Hall Inc., 1952, pp. 850.

4. Beattie BR, Taylor CR, Watts MJ. The Economics of Production, 2nd edn. Malabar, Florida: Krieger Publishing Company, 2009, pp. 299.

5. Häsler BN, Economic assessment of veterinary surveillance programmes that are part of the national control plan of Switzerland (thesis). London, UK: University of London, Royal Veterinary College, 2011, $235 \mathrm{pp}$.

6. Thrusfield M. Veterinary Epidemiology, 3rd edn. Oxford: Blackwell Publishing Science, 2005, pp. 137-144, 364-367.

7. Hicks JR. The Theory of Wages. London: Macmillan, 1932, pp. 117.
8. Heathfield DF, Wibe S. An Introduction to Cost and Production Functions. Basingstoke and London: Macmillan Education Ltd, 1987, pp. 58-60.

9. Howe KS, Christiansen KH. The state of animal health economics: a review. In: Reid SWJ, Menzies FD, Russell AM, eds. Proceedings of the Society for Veterinary Epidemiology and Preventive Medicine. Martigny: Society for Veterinary Epidemiology and Preventive Medicine, 2004, pp. 157-158.

10. Rushton J, Viscara R, Otte J, McCleod A, Taylor N. Animal health economics - where have we come from and where do we go next. $C A B$ Reviews: Perspectives in Agriculture, Veterinary Science, Nutrition and Natural Resources 2007; 031 : 1-10.

11. Rich KM, Perry BD. The economic and poverty impacts of animal diseases in developing countries: new roles, new demands for economics and epidemiology. Preventive Veterinary Medicine 2011; 101: 133-147.

12. Rich K. New methods for integrated models of animal disease control. Paper presented at the American Agricultural Economics Association Annual Meeting, Portland, Oregon, 29 July-1 August 2007 (http:// ageconsearch.umn.edu/bitstream/9701/1/sp07ri03.pdf).

13. Häsler B, Howe KS, Di Labio E, Schwermer H, Stärk KDC. Economic evaluation of the surveillance and intervention programme for bluetongue virus serotype 8 in Switzerland. Preventive Veterinary Medicine 2012; 103: 93-111.

14. Häsler B, Howe KS, Presi P, Stärk KDC. An economic model to evaluate the mitigation programme for bovine viral diarrhoea in Switzerland. Preventive Veterinary Medicine. Published online: 6 March 2012. doi:10.1016/ j.prevetmed.2011.12.010.

15. Häsler B, et al. A qualitative approach to measure the effectiveness of active avian influenza virus surveillance with respect to its cost: a case study from Switzerland. Preventive Veterinary Medicine. Published online: 31 January 2012. doi:10.1016/j.prevetmed.2011.12.010

16. Mishan EJ, Quah E. Cost-Benefit Analysis, 5th edn. London, New York: Routledge, 2007, pp. 329.

17. Fujiwara D, Campbell R. Valuation techniques for social cost-benefit analysis: stated preference, revealed preference and subjective well-being approaches-a discussion of the current issues. London: HM Treasury and Department for Work and Pensions, 2011, pp. 76.

18. Rushton J. The Economics of Animal Health and Production, Oxford: CABI International, 2009, pp. 124-127.

19. McInerney JP, Howe KS, Schepers JA. Cost-benefit analysis of disease control programmes. In: $A$ Framework and Methodology for the Economic Analysis of Disease in Farm Livestock, Report of a Research Project, Ref. CSA 873, funded by the Ministry of Agriculture, Fisheries and Food. University of Exeter: Agricultural Economics Unit, 1990, pp. 39-44.

20. McInerney JP. Cost-benefit analysis of livestock disease: a simplified look at its economic foundations. In: Martin SW, ed. Proceedings of the 6th ISVEE Symposium. Ottawa: International Symposium on Veterinary Epidemiology and Economics, 1991, pp. 149-153. 
21. Tisdell C. Assessing the approach to cost-benefi analysis of controlling livestock diseases of McInerney and Others. Research Papers and Reports in Animal Health Economics, No. 3. Brisbane: Department of Economics, University of Queensland, 1995. pp. 21.

22. Brown M. The Theory and Empirical Analysis of
Production. Studies in Income and Wealth, volume 31. New York: National Bureau of Economic Research, distributed by Columbia University Press, 1967, pp. 515.

23. Heady EO, Dillon JL. Agricultural Production Functions. Ames, Iowa: Iowa State University Press, 1961, pp. 667. 